\title{
Scaphoid-Lunate Joint
}

National Cancer Institute

\section{Source}

National Cancer Institute. Scaphoid-Lunate Joint. NCI Thesaurus. Code C142314.

The articulation of the scaphoid and lunate bones in the wrist. 